\title{
Error Detection in Turbo Decoding using Neural Network
}

\author{
S.Bhavanisankari, G.T.Bharathy, T.Tamilselvi
}

\begin{abstract}
In this paper reduction of errors in turbo decoding is done using neural network. Turbo codes was one of the first thriving attempt for obtaining error correcting performance in the vicinity of the theoretical Shannon bound of $-1.6 \mathrm{db}$. Parallel concatenated encoding and iterative decoding are the two techniques available for constructing turbo codes. Decrease in Eb/No necessary to get a desired bit-error rate (BER) is achieved for every iteration in turbo decoding. But the improvement in Eb/No decreases for each iteration. From the turbo encoder, the output is taken and this is added with noise, when transmitting through the channel. The noisy data is fed as an input to the neural network. The neural network is trained for getting the desired target. The desired target is the encoded data. The turbo decoder decodes the output of neural network. The neural network help to reduce the number of errors. Bit error rate of turbo decoder trained using neural network is less than the bit error rate of turbo decoder without training.
\end{abstract}

Index Terms: Turbo Codes, Neural Networks, Encoder, Decoder, Bit Error Rate.

\section{INTRODUCTION}

The Wireless communication is a quickly rising division of communication industry, with the prospective to afford elevated speed superior quality information exchange among portable devices positioned anywhere in the universe. Communication system uses digital communication scheme for sending and receiving data. Claude Berrou and Alain Glaviex had invented a digital coding scheme that could offer almost error-free communications at better data rates and transmitting power efficiency. Their error correcting coding format, which was named as turbo codes, has revolutionized error-correcting coding [4].

Block coding schemes separates bit stream into non-overlapping blocks and the coding is performed for each block independently. The encoder receives a $\mathrm{k}$ bit message block and create and $\mathrm{n}$ bit code word [5]. Thus code words are formed on block-by-block basis. In convolutional code, the channel encoder receives message bits as a uninterrupted sequence and thereby generates a uninterrupted sequence of encoded bits at superior rate. Coding of successive information blocks makes the block coding schemes memory less.

Convolutional codes are a popular class of coders with memory [5].Turbo coding is a novel and very commanding

Revised Manuscript Received on October 12, 2019

Ms.S.Bhavanisankari, Associate Professor, Department of ECE, Anna University/ Jerusalem College of Engineering/ Chennai, India.

Ms.G.T.Bharathy, Associate Professor, Department of ECE, Anna University/ Jerusalem College of Engineering/ Chennai, India.

Ms.T.Tamilselvi, Associate Professor, Department of ECE, Anna University/ Jerusalem College of Engineering/ Chennai, India. error control procedure. Turbo codes can function at $0.1 \mathrm{db}$ from Shannon capacity limit out performing all supplementary coding technique available in recent days [4].

\section{TURBO ENCODER}

Convolutional coding is utilized for a continuous input stream, as a substitution to the block codes for broadcasting over a noisy channel. Infinite impulse response is generated by an encoder with a feedback loop.

In a systematic code, the outputs serves as an input itself [4]. Binary non-recursive non-systematic convolutional encoder is repeatedly used. The output is evaluated with the blend of the current input and a limited number of previous inputs [4].

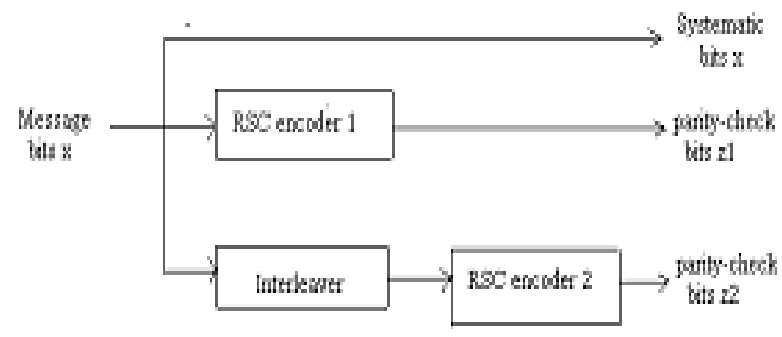

Figure1: Turbo Encoder

A convolutional encoder is represented as $(\mathrm{n}, \mathrm{k}, \mathrm{K})$ format, in which $\mathrm{n}$ is the encoder output, $\mathrm{k}$ is the encoder input, $\mathrm{K}$ is constraint length, which is the number of memory elements +1 . The output $\mathrm{n}$ of the encoder at any specified moment depends on the inputs $\mathrm{k}$ and as well as on the past inputs $\mathrm{m}$. The encoder is a combination of an $\mathrm{m}$ stage shift register, modulo- 2 adders and a multiplexer.

The recursive systematic convolutional encoder is derived with the help of non recursive non-systematic convolutional encoder in which one of its encoded output if fed back as an input. The convolutional encoder is denoted by the generator sequences $\mathrm{G}=[\mathrm{g} 1, \mathrm{~g} 2]$.

In Turbo Codes, between the two component encoders an interleaver is utilized. The interleaver offers unpredictability to an input sequences and also raises the weight of the code words. An interleaver alters the sequence of symbols by a specific approach. The most critical part in turbo code is the interleaver [4].

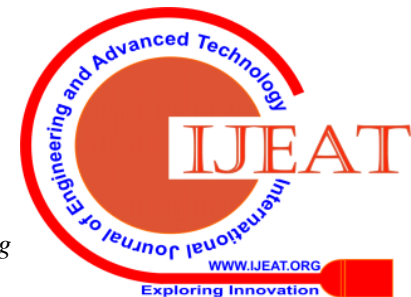




\section{TURBO DECODER}

The decoder consists of two log-map decoders in a parallel-concatenated scheme [4]. Noisy versions of the $\mathrm{k}^{\text {th }}$ systematic bit $X_{k}$ are given as inputs to decoder 1 . The redundant information $\mathrm{y}_{1 \mathrm{k}}$ and a third 'a priori' input $\mathrm{z}_{1 \mathrm{k}}$, which accepts feedback from the decoder2. Decoder 2 receives information from decoder 1 , which cannot be derived from its own redundant input $\mathrm{z}_{2 \mathrm{k}}$.

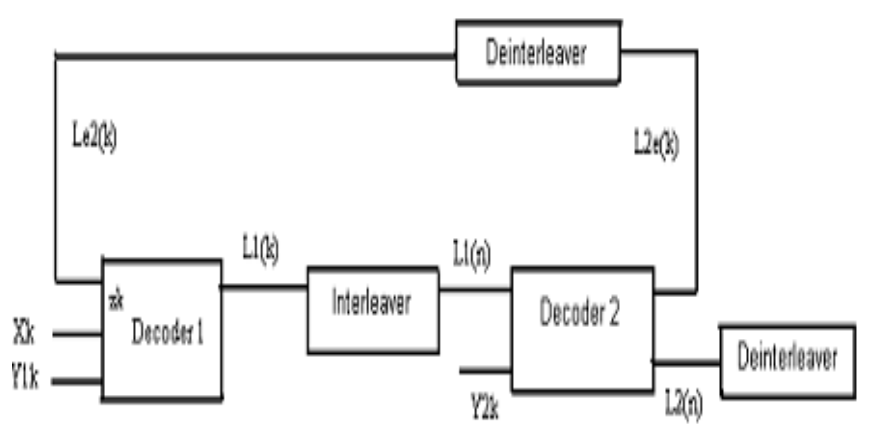

Figure 2: Turbo Decoder

The interleaver makes sure that information fed to a decoder appears independent of the information generated by its neighbor [4]. Thus the information exchanged can be used to improve the decision of each bit being encoded.

An imperative prerequisite for iterative decoding is the capability to estimate the soft decision values for every data bit. The soft decision statistics are universally represented by a log likelihood ratio (LLR).

\section{NEURAL NETWORK}

An artificial neural network (ANN) works similar to biological nervous systems. It comprises of a massive number of extremely interrelated processing elements (neurons) which solves precise troubles. An ANN is developed for precise purpose, such as recognition of pattern, classification of data, with the help of a learning process.

\section{A. Neural Network Model}

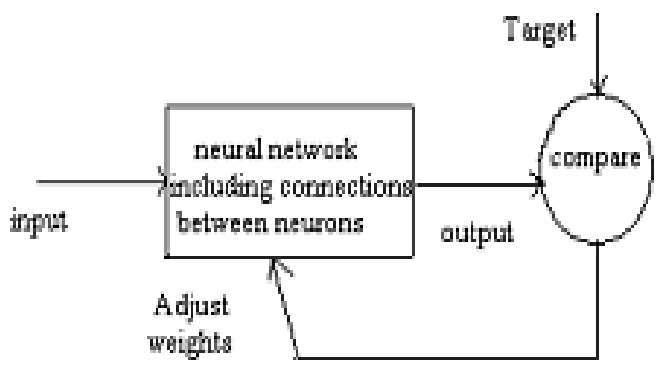

Figure 3: Neural Network Model

Neural networks comprises of uncomplicated elements functioning in parallel which are motivated by biological nervous systems. Neural network could be educated to carry out a specific role by varying the values of the weights linking the elements. Commonly adjustment or the training of neural network is done to get a specific target output, for a particular input. In this supervised learning, huge amount of input/target pairs are utilized for training a network [3]. Batch training of a network is done by changing the weight and bias, considering the complete range of input vectors.

\section{B. Training Neural Networks}

The network can be educated for function approximation (nonlinear regression), pattern association, or pattern classification. The network inputs $\mathrm{p}$ and target outputs $\mathrm{t}$ is essential for the course of action of training. The network performance function is minimized by the proper iterative adjustments of weights and biases of a network. The important performance characteristics of a feed forward networks is mean square error. The gradient of the performance function is used by the training algorithm to adjust the weights. Back propagation method is utilized for evaluating the gradient, by computing the network in the reverse order.

Steps involved in the Training of a network:

1. The training pair is selected and applied as input vector to the network input.

2. Network output is evaluated with the help of forward pass. Evaluation of network output is achieved with the help of a feed forward process and appliance of an activation function in the network for every layer.

3. The difference between the network output and the desired target value is compared.

4. Vary the network weights in such a way to reduce the error.

\section{Backpropagation Training}

The easiest realization of backpropagation learning, improvises the network weights and biases in the direction by which the performance function reduces most quickly - the negative of the gradient. The gradient descent algorithm can be realized using the following two methods: Incremental mode, Batch mode. The gradient is evaluated and the weights are rationalized after the application of every input to the network, in the incremental mode. In the batch mode the entire inputs are given as input to the network prior to the weight updation. The gradients evaluated at every training are summed to establish the alteration required for the weights and biases [3].

\section{DESIGN METHODOLOGY}




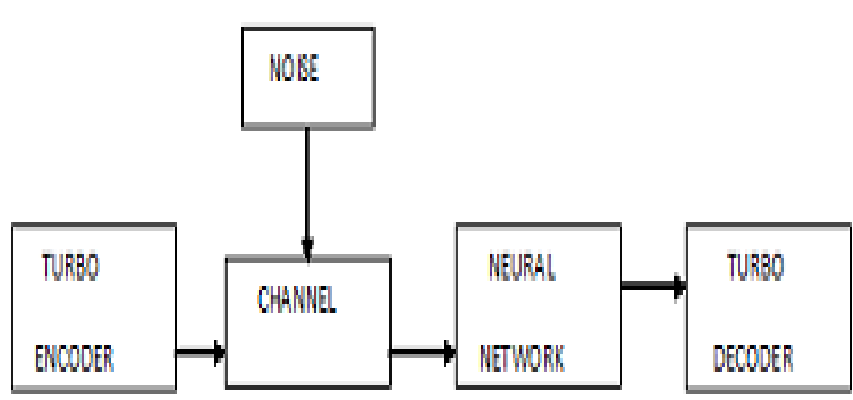

Figure 4: Functional Block Diagram

From the turbo encoder, the output is taken and this is added with noise, when transmitting through the channel. The noisy data is fed to the neural network. The neural network is trained to get the desired target. The desired target is the encoded data. The turbo decoder decodes the output of neural network. The neural network help to reduce the number of errors. Bit error rate of turbo decoder trained using neural network is less than the turbo decoder without training. Fig.5 shows reduction of errors in turbo decoding done using neural network.

\section{SIMULATION RESULTS}

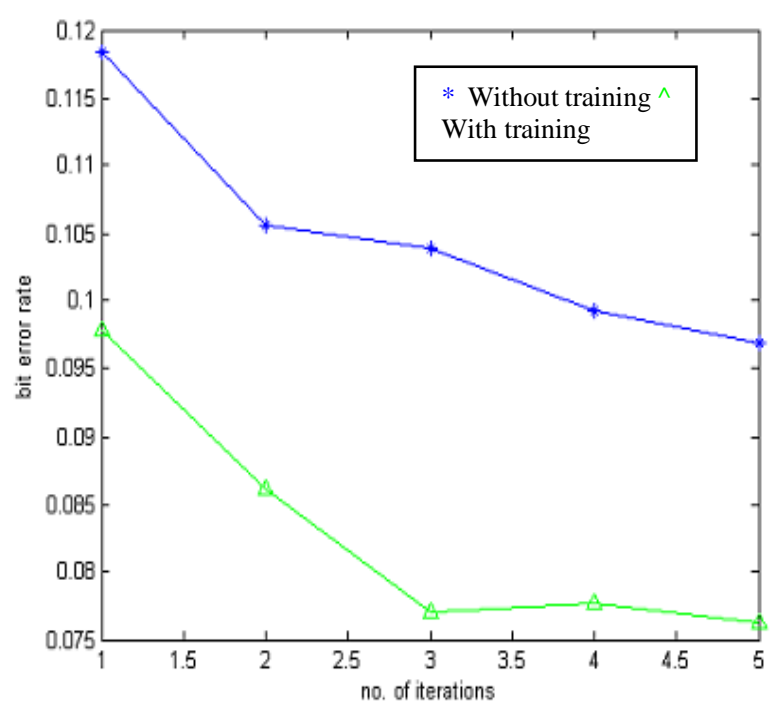

Figure 5: Training With Neural Network

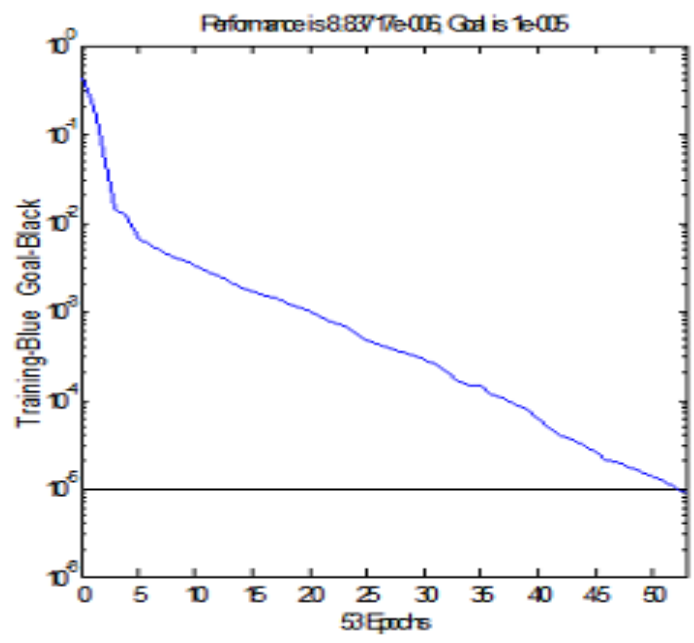

Fig.6: Bit Error Rate Versus Number of Iterations

Fig.6 shows training with neural networks. The neural networks trains 10 sets of 500 bits of noisy data that fed to the neural network as input. The noisy data is the encoded data added with noise, while transmitting through the channel. The desired target is the encoded data. The neural network trains the noisy data to reach the desired performance. The neural network helps to reduce the number of errors. In fig.6 the green line with ${ }^{\wedge}$ represents number of iterations verses bit error rate of a turbo decoder trained using neural network. The blue line with $*$ represents the number of iterations verses bit error rate of a Turbo decoder without training. The fig.6 shows that the bit error rate of turbo decoder trained using neural network is less than the turbo decoder without training.

\section{CONCLUSION}

In this paper, the reduction of errors in turbo decoding is done using neural network. From the turbo encoder, the output is taken and this is added with noise, when transmitting through the channel. The noisy data is fed to the neural network. The neural network is trained to get the desired target. The desired target is the encoded data. The turbo decoder decodes the output of neural network. The neural network helps to reduce the number of errors. Bit error rate of turbo decoder trained using neural network is less than the turbo decoder without training.

\section{REFERENCES}

1. " Techniques for early stopping and error detection in turbo decoding" in IEEE transaction of communication, vol51, no.10, October 2003.

2. "A neural network for predicting decoder error in turbo decoders" in IEE communication letters, vol 3,no.5, may1999.

3. "The design and performance of neural network for predicting turbo decoding error with application to hybrid ARQ protocols" in IEEE transactions of communication, vol 48, april 2000.

4. " Near Shannon limit error correcting coding and decoding in Proc. IEEE Conf. Communications, 1993.

5. J. Hagenauer, E. Offer, and L. Papke, "Iterative decoding of binary block and convolutional codes," IEEE Trans.Inform. Theory, vol. 42,pp. 429-445 Mar. 1996.

6. R. Y. Shao, S. Lin, and M. P. C.Fossorier, "Two simple stopping criteria for turbo decoding," IEEE 
Trans. Commun., vol47, pp. 1117-1120,Aug.1999.

7. A. Ambroze, G.Wade, and MTomlinson, "Practical aspects of iterative decoding," Elect. Eng. Proc.-Commun., vol. 147, pp.69-74, Apr.2000.

8. Y. Wu, B. D. Woerner, and W. J. Ebel "A simple stopping criterion for turbodecoding," IEEE Commun. Lett., vol. 4,pp.258-260, Aug. 2000.

9. Benedetto, S., et al., "Soft Output Decoding Algorithm in Iterative Decoding of Turbo Codes," TDA Progress Report 42-124, Jet Propulsion Laboratory, Pasadena, California, Vol. 18 pp. 63-87, February 15, 2017

10. Thomas Jerkovits, balazs Matuz "Turbo Code Design For Short Blocks", Proceedings IEEE, International Conference on Communications, Vol. 19 Feb. 2016

\section{AUTHORS PROFILE}

Ms.S.Bhavani Sankari was born in India in the year 1978. She completed B.E degree in Electronics and Communication Engineering from Thanthai Periyar Government College of Engineering, Madras University, India in the year 1995 and M.E degree in Industrial Electronics from Sri Jeyachamarajendra College of Engineering, Visveswaraya University, India in the year 2003. She is now working as an Associate Professor in Jerusalem College of Engineering, Dept. of Electronics and Communication Engineering, Chennai. She is a life member in ISTE. Her research interest is Communication, Signal Processing.

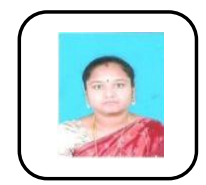

Ms.G.T.Bharathy was born in India in the year 1979. She completed B.E degree in Electronics and Communication Engineering from Easwari Engineering College, Chennai, Madras University, India in the year 2000 and M.E degree in Communication Systems from Shri Venkateshwara College of Engineering, Chennai, Anna University, India in the year 2005. She is now working as Associate Professor in Jerusalem College of Engineering, Dept. of Electronics and Communication Engineering, Chennai. She is a life member in ISTE. She has worked as Lecturer in the department of ECE in Anand Institute of Higher Technology and Prince Shri Venkateshwara Padmavathi College of Engineering, Chennai. Her research interest is RF \& Microwave circuits, and Communication systems.

Ms.T.Tamilselvi was born in India in the year 1978. She completed B.E degree in Electronics and Communication Engineering from Adhiparasakthi Engineering College, Madras University, India in the year 2000 and M.E degree in Embedded System Technologies from College of Engineering, Guindy (CEG Main Campus), Anna University, India in the year 2006. She is now working as Associate Professor in Jerusalem College of Engineering, Dept. of Electronics and Communication Engineering, Chennai. She is a life member in ISTE. Her research interest is VLSI and Embedded design. 